\title{
A NEW CONCEPT FOR RADIONUCLIDE GENERATOR SYSTEMS
}

\author{
Bontchev G. D. ${ }^{1}$, S. G. Bontcheva ${ }^{2}$ \\ ${ }^{1}$ Dept. of Pharmacology, chemistry, biochemistry and biology, ${ }^{2}$ Dept. of Physics and biophysics, \\ Medical University "Prof. P. Stoyanov" - Varna
}

Reviewed by: Assoc. Prof. M. Stancheva

\begin{abstract}
Radiodiagnostic methods of nuclear medicine use short-lived isotopes for variety of imaging purposes. In medical centers these isotopes are produced commonly by special equipment, as known as "radionuclide generators". The radionuclide generated by them can be periodically extracted and, after simple chemical processing, used for appropriate medical application. In developed and commercially available radionuclide generators, the separation and extraction of isotopes is set up on the principles of forced column chromatography, i.e. by means of positive (or negative) pressure-driven elution. Although these sorption-type generators work fine and, in general, accomplish most of the requirements for medical application, yet another principle of generator's construction appears to be also possible. In this paper a new concept for developing a generator system, in which the separation and extraction of radionuclides is carried out on an electrophoretic basis is discussed. Details such as construction possibility, theoretical motivation as well as expected effectiveness are considered. Some possible advantages, concerning radiopharmaceutical usage of proposed electrophoretic generators are outlined.
\end{abstract}

Key words: radionuclide generators, electrophoresis, nuclear medicine

\section{INTRODUCTION}

Radioisotopes are successfully used in nuclear medicine for many years. Generally, three main areas of their application could be clearly recognized: radioimmuno-assay, radiotherapy and radiodiagnostic.

Radioimmuno-assay (RIA) uses variety of radionuclides (Table 1) for different in-vitro biochemical analyses, based mostly on radiolabeling of biologically active molecules for tracing and detecting. It is highly popular, simple and effective technique: about 15 million radioimmuno-assays procedures are undertaken in Europe each year (7). Most important nuclide appears to be I-125, but role of beta emitters as $\mathrm{H}-3$ and C-14 increases from some years now, because radiolabeling with hydrogen/carbon avoids introduction of heavy hetero-atom into sensitive biomolecules.

Radiotherapy exploits the fact that rapidly dividing cells are particularly sensitive to damage by radiation. For this reason, some cancerous growths can be controlled or eliminated by irradiating the area containing the growth. Extracorporealirradiation (focused or hemi-body) can be managed by high energy gamma-beam from shielded and

Address for correspondence:

G. Bontchev, Dept. of Pharmacology, Chemistry, Biochemistry and Biology, Faculty of Dental Medicine, Medical University "Prof. P. Stoyanov" - Varna, 55 Marin Drinov Str., 9002-Varna, Bulgaria e-mail: bontchev@mu-varna.bg collimated Co-60 source. Nowadays linear accelerators as a source of high-energy X-rays are preferred for this purpose,

Table 1. Major isotopes used for radioimmuno-assay (RIA) /bold: most important/.

\begin{tabular}{||c|c|c||}
\hline \hline H-3 & Ca-47 & In-111 \\
\hline C-14 & Co-57 & In-113m \\
\hline Na-22 & Co-58 & I-125 \\
\hline P-32 & Fe-59 & I-131 \\
\hline S-35 & Ga-67 & \\
\hline
\end{tabular}

Table 2. Major isotopes used for traditional radiotherapy /bold: most important/.

\begin{tabular}{||c|c|c||}
\hline P-32 & Sn-117m & Ho-166 \\
\hline Co-60 & I-125 & Er-169 \\
\hline Cu-67 & I-131 & Yb-175 \\
\hline Sr-89 & Pm-149 & Lu-177 \\
\hline Y-90 & Sm-153 & Re-186 \\
\hline Pd-103 & Dy-165 & Re-188 \\
\hline In-111 & Dy-166 & Ir-192 \\
\hline \hline
\end{tabular}


though. Intracorporeal irradiation is administered by many forms - drugs, seed implants, needles, etc. Usually long-lived gamma and beta emitters are used (Table 2), although recently developed Targeted Alpha Therapy (TAT) appears to be very promising, especially for the control of dispersed cancers (Table 3).

Table 3. Major isotopes used for Targeted Alpha Therapy (TAT) /bold: most important/.

\begin{tabular}{||c|c|c|}
\hline \hline Tb-149 & Bi-213 & Fm-255 \\
\hline At-211 & Ra-223 & \\
\hline Bi-212 & Ac-225 & \\
\hline \hline
\end{tabular}

Radiodiagnostic uses the gamma rays emitted by some nuclides to make internal body organs and tissue visible in a manner similar to the way X-rays provide images of bones. The difference is that in radiodiagnostic procedures the source of radiation is located within the body. The nuclides (Table 4), linked to a specific chemical compound and introduced in patients (by injection, inhalation or orally) allows to screen the corresponding body zones in which this particular compound is naturally accumulated. The detector, known as "gamma camera", builds up a $3 \mathrm{D}$ (or 3+1 D) image of the points from which radiation is emitted. This summarizes the Single Photon Emission Computed Tomography (SPECT).

Table 4. Major isotopes used for Single Photon Emission Computed Tomography (SPECT) /bold: most important/.

\begin{tabular}{||c|c|c||}
\hline Na-24 & Gd-68 & I-125 \\
\hline K-42 & Se-75 & I-131 \\
\hline Cr-51 & Kr-81m & Xe-133 \\
\hline Fe-59 & Tc-99m & Yb-169 \\
\hline Cu-64 & In-111 & Tl-201 \\
\hline
\end{tabular}

A more recent development is Positron Emission Tomography (PET) which is a more precise and sophisticated technique using cyclotron-produced isotopes (Table 5). It has been proven that PET is the most accurate non-invasive method of detecting and evaluating cancers. New procedures (PETCT) combine PET with computer assisted $\mathrm{X}$-ray tomography (CT), enabling $30 \%$ better diagnosis than with traditional gamma camera alone (7).

Radiopharmaceutics used in nuclear medicine for radiodiagnostic purposes (scintigraphy, screening, tomography, radiolabeling etc.) contain an appropriate, usually short-lived isotope in suitable chemical form. Isotopes of interest can be produced via nuclear reaction at cyclotrons and nuclear reactors, but, due to his extremely short half-live period (usually minutes or hours), it is definitely inconvenient - and more often impossible - to be transported to hospitals for application.

Table 5. Major isotopes used for Positron Emission Tomography (PET) /bold: most important/.

\begin{tabular}{||c|c|c||}
\hline C-11 & F-18 & Ga-68 \\
\hline N-13 & K-38 & Rb-82 \\
\hline O-15 & Cu-62 & \\
\hline
\end{tabular}

To get this problem solved the radionuclide generator systems has been designed and successfully used for many years now. These portable devices ("Cows") produce the isotopes needed via spontaneous radioactive decay of an appropriate, long-lived radionuclide ("Mother"), fixed onto inert inorganic adsorbent. Accumulated short-lived isotope of interest ("Daughter") could be periodically separated from mother nuclide by means of chemical extraction on chromatographic basis, e.g. elution by suitable solvent, usually - physiological solution ("To Milk the Cow"). Most important radionuclide generators used in nuclear medicine are listed in Table 6.

\section{MATERIALS AND METHODS}

An automatized device for horizontal zone electrophoresis in a free electrolyte was used for measuring ion mobility (3). The equipment as well as the methodic used allows determining the electrophoretic ion mobility with an unique relative error less than $0,5 \%(6)$. All of the experiments were done at the temperature $(25,00 \pm 0,05)^{\circ} \mathrm{C}$ and gradient of the electric field kept constant near $10 \mathrm{~V} \cdot \mathrm{cm}^{-1}$. pH of the solutions was measured with an accuracy of $0,02 \mathrm{pH}$-units. Isotopes used in electromigration runs described in the present paper: ${ }^{111} \mathrm{In}\left(\mathrm{T}_{1 / 2}=2,83 \mathrm{~d}\right.$; $\mathrm{E}_{\mathrm{r}}: 171,3 \mathrm{keV}(90,3 \%)$, $245,4 \mathrm{keV}(94,0 \%)),{ }^{11 \mathrm{~m}} \mathrm{Cd}\left(\mathrm{T}_{1 / 2}=49 \mathrm{~m} ; \mathrm{E}_{\mathrm{r}}: 150,8 \mathrm{keV}\right.$ (29,1\%), 245,4 keV (94,0\%)), ${ }^{88} \mathrm{Zr}\left(\mathrm{T}_{1 / 2}=83,4 \mathrm{~d} ; \mathrm{E}_{\mathrm{r}}: 392,9\right.$ $\mathrm{keV}(97,3 \%))$ and ${ }^{175} \mathrm{Hf}\left(\mathrm{T}_{1 / 2}=70 \mathrm{~d} ; \mathrm{E}_{\mathrm{r}}: 89,4 \mathrm{keV}(2,4 \%)\right.$, $343,4 \mathrm{keV}(84 \%), 433,0 \mathrm{keV}(1,4 \%))$ were produced at U-200 cyclotron (Flerov Laboratory of Nuclear Reactions, Dubna, Russia) via $(\alpha, x n)$ reactions using $30-35 \mathrm{MeV}$ $\alpha$-particles. Radionuclide species were concentrated and purified by means of ion-exchange chromatography $(1,4)$. Chemical reagents: $\mathrm{HClO}_{4}, \mathrm{HNO}_{3}, \mathrm{NaClO}_{4}, \mathrm{KNO}_{3}, \mathrm{NaOH}$, $\mathrm{KOH}, \mathrm{DTPA}$ (diethilenetriaminepentaacetic acid) were of p.f.a. grade (in some cases - Merck $^{\circledR}$ Suprapur $^{\circledR}$ ). All solutions were prepared using bidistilled and deionized water.

\section{RESULTS AND DISCUSSION}

Technically speaking, radionuclide generator systems are portable chemical separators. Mother isotope generates daughter isotope via spontaneous radioactive decay, forming in such a way a homogenous mixture. The main generator's role is to separate obtained chemical entities; that is, 
to set apart daughter from mother nuclides. The separation procedure must be simple and effective one, as well as should ensure that mother isotope will remain within generator's container. The generator's construction itself must guarantee an efficient shielding from mother and daughter radioactive radiation.
When radiochemical equilibrium between the mother and daughter radioisotopes is established, the extraction procedure could be started: an appropriate eluant, e.g. pH-adjusted $0,9 \% \mathrm{NaCl}$, is forced trough the system by means of positive pressure (syringe, attached to inlet) or negative pressure (evacuated vial, attached to outlet). Thus, daughter

Table 6. Common radionuclide generators used in nuclear medicine.

\begin{tabular}{|c|c|c|c|c|c|c|}
\hline mother & half life & decay mode & daughter & half life & decay mode & terminator \\
\hline Si-32 & $153(19) \mathrm{y}$ & $\beta$ & P-32 & $14,262(14) d$ & $\beta$ & S-32 (st) \\
\hline Zn-62 & $9,186(13) \mathrm{h}$ & $\mathrm{EC}$ & $\mathrm{Cu}-62$ & $9,67(3) \mathrm{m}$ & $\mathrm{EC}$ & Ni-62 (st) \\
\hline $\mathrm{Ni}-66$ & $54,6(3) \mathrm{h}$ & $\beta$ & $\mathrm{Cu}-66$ & $5,120(14) \mathrm{m}$ & $\beta$ & Zn-66 (st) \\
\hline Ge-68 & $270,95(16) d$ & $\mathrm{EC}$ & Ga-68 & $67,71(9) \mathrm{m}$ & $\mathrm{EC}$ & $\mathrm{Zn}-68$ (st) \\
\hline Rb-81 & $4,570(4) h$ & $\mathrm{EC}$ & $\mathrm{Kr}-81 \mathrm{~m}$ & $13,10(3) \mathrm{s}$ & IT & $\mathrm{Kr}-81\left(^{*}\right)$ \\
\hline Sr-82 & $25,55(15) d$ & $\mathrm{EC}$ & Rb-82 & $1,273(2) \mathrm{m}$ & $\mathrm{EC}$ & $\mathrm{Kr}-82(\mathrm{st})$ \\
\hline Sr-90 & $28,90(3) y$ & $\beta$ & Y-90 & $64,053(20) \mathrm{h}$ & $\beta$ & $\mathrm{Zr}-90$ (st) \\
\hline Mo-99 & $65,94(1) h$ & $\beta$ & Tc-99m & $6,0058(12) \mathrm{h}$ & IT & Tc-99 (*) \\
\hline Sn-113 & $115,09(3) \mathrm{d}$ & $\mathrm{EC}$ & In-113m & $99,476(23) \mathrm{m}$ & IT & In-113 (st) \\
\hline Te-132 & $3,204(13) d$ & $\beta$ & I-132 & $2,295(13) \mathrm{h}$ & $\beta$ & Xe-132 (st) \\
\hline W-188 & $69,78(5) \mathrm{d}$ & $\beta$ & Re-188 & $17,003(3) \mathrm{h}$ & $\beta$ & Os-188 (st) \\
\hline Ac-225 & $10,0(1) \mathrm{d}$ & $\alpha$ & $\begin{array}{c}{[\text { Fr-221, } \alpha \downarrow} \\
{[\text { At-217, } \downarrow] \downarrow} \\
\text { Bi-213 }\end{array}$ & $45,59(6) \mathrm{m}$ & $\beta$ & $\begin{array}{c}{[\mathrm{Po}-213, \alpha] \downarrow} \\
{[\mathrm{Pb}-209, \beta] \downarrow} \\
\text { Bi-209 (st) }\end{array}$ \\
\hline Th-227 & $18,68(9) d$ & $\alpha$ & Ra-223 & $11,43(5) d$ & $\alpha$ & $\begin{array}{c}{[\mathrm{Rn}-219, \alpha] \downarrow} \\
{[\mathrm{Po}-215, \alpha] \downarrow} \\
{[\mathrm{Pb}-211, \beta] \downarrow} \\
{[\mathrm{Bi}-211, \alpha] \downarrow} \\
{[\mathrm{Tl}-207, \beta] \downarrow} \\
\mathrm{Pb}-207 \text { (st) }\end{array}$ \\
\hline
\end{tabular}

DECAY MODES:

$$
\begin{aligned}
& \alpha=\text { alpha-decay } \\
& \beta=\text { beta-decay } \\
& \mathrm{EC}=\text { electron capture } \\
& \mathrm{IT}=\text { isomeric transition }
\end{aligned}
$$

\section{SYMBOLS:}

$$
\begin{aligned}
& (\text { st })=\text { stabile } \\
& (*)=\text { half-life }>1.10^{5} \mathrm{y} \\
& \downarrow=\text { decays further }
\end{aligned}
$$

\section{Principles of existing generators}

At present time most of the generator systems, especially one's used in nuclear medicine, are based on principles of chromatography. They feature a plane construction (Fig. 1) as well as simple maintenance.

Initially, a specific amount of mother radionuclide is fixed onto inert (and usually) inorganic adsorbent, e.g. $\mathrm{Al}_{2} \mathrm{O}_{3}$, and the system is capsulated within a shielded container. nuclide is washed out from the system and mixed with a specific kit (a chelating agent + buffer solution) to form as a result an injection-ready tracer. Mother nuclide remains within the generator and, in relatively short period of time, accumulates fresh amount of daughter isotope. In described procedure the separation of daughter from mother nuclides is taking place due to their different sorption behavior; that is why the generators organized on such principle are called "sorption-type" or "chromatographic type".

\section{Principles of new generators}

During investigation on electrophoretic methods for separation, a new radionuclide generator concept has been raised. Proposed generators are based on principles of electrophoresis. Avoiding macroscopic fluid fluxes during work cycle ensures even further simplification of their construction (Fig. 2).

Initially, a specific amount of mother radionuclide is dissolved in an appropriate electrolyte (chelating agent + buffer solution) and is capsulated together with two elec- 
trodes within a shielded container. When radiochemical equilibrium between the mother and daughter radioisotopes is established, the extraction procedure could be started: a high voltage current is switched on. Daughter nuclide migrates toward one of the electrodes and could be collected as already formed tracer. Mother nuclide migrates

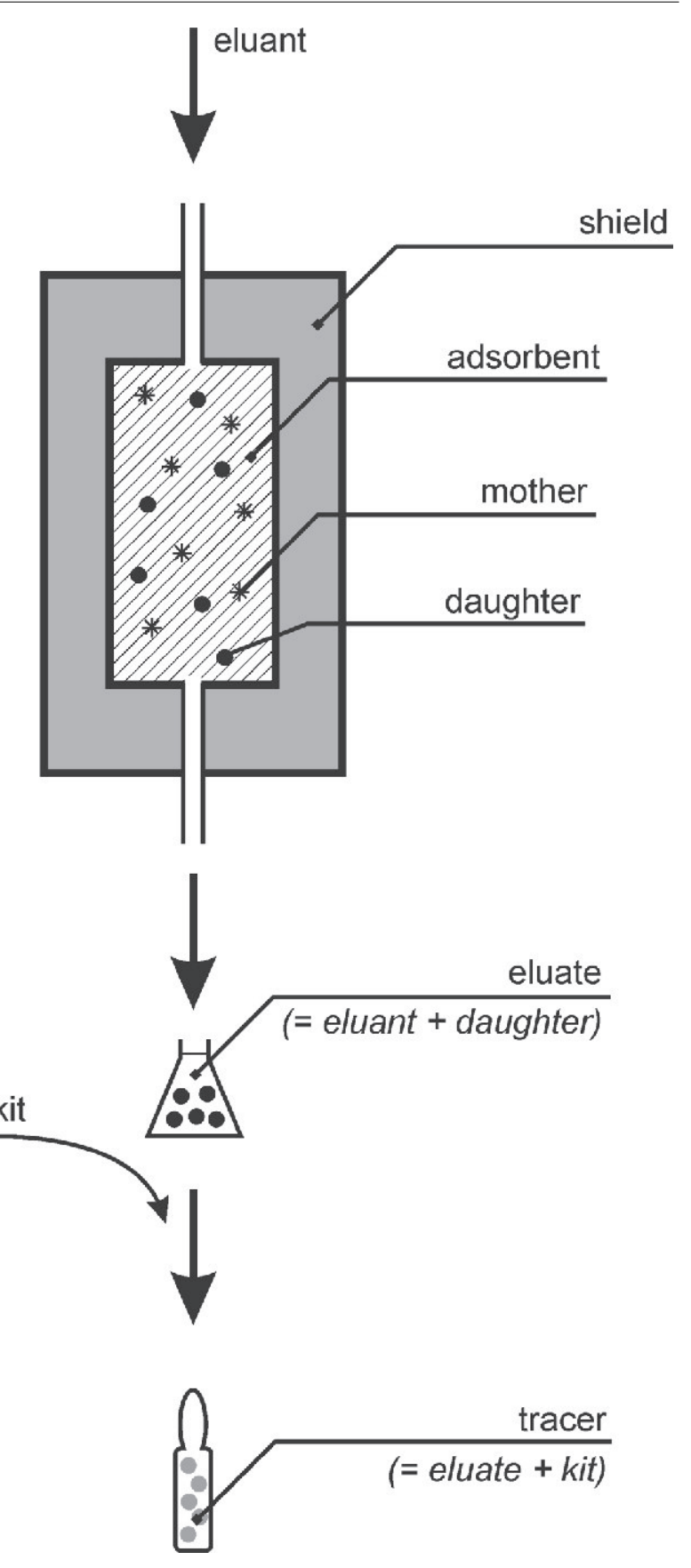

Fig. 1. Schematic construction of well-known radionuclide generator (chromatographic type)

toward the other electrode, thus remains within the generator and, in relatively short period of time, accumulates fresh amount of daughter isotope. In described procedure the separation of daughter from mother nuclides is taking place due to their opposite electrophoretic behavior; that is why the generators organized on such principle should be called "mobility-type" or "electrophoretic type".

\section{Theoretical basis of new generators}

However it should be boldly underlined that generators of electrophoretic type will properly work if only the electrolyte's parameters are carefully chosen. That is, the experimental conditions must guarantee that mother nuclide's and daughter nuclide's chemical forms electromigrate in an opposite directions. Such a demand may look extremely complicated one but, in fact, it could be easily arranged in most cases, even when chemically very close species as $\mathrm{Zr}$ and $\mathrm{Hf}$ are concerned (5).

Kits used in nuclear medicine usually are chelating agents and represents a weak polyvalent acids of type $\mathrm{H}_{\mathrm{n}} \mathrm{Y}$ (e.g. EDTA, DTPA, NTA etc.). In aqueous solution they undergo multi-step dissociation, which we could express in summarized form as follows:

(1) $\mathrm{H}_{\mathrm{n}} \mathrm{Y} \rightleftharpoons \mathrm{nH}^{+}+\mathrm{Y}^{\mathrm{n}-}$.

Metal cations (e.g. mother nuclide) $\mathrm{Me}^{\mathrm{m}+}$ introduced in solution are engaged in complex formation process:

(2) $\mathrm{Me}^{\mathrm{m}+}+\mathrm{Y}^{\mathrm{n}-} \rightleftarrows[\mathrm{MeY}]^{(\mathrm{n}-\mathrm{m})-}$.

Conjugated chemical equilibriums (1) - (2) actually describe a competition between two cations $\left(\mathrm{H}^{+}\right.$and $\left.\mathrm{Me}^{\mathrm{m}+}\right)$ for a ligand $\left(\mathrm{Y}^{\mathrm{n}}\right)$. This competition obviously could be manipulated by varying the proton concentration, i.e. adjusting $\mathrm{pH}$ of the solution. In other words, we do have a possibility to force the nuclide into positive- or negative-charged ionic form. If a second metal ion (e.g. daughter nuclide) $\mathrm{M}^{\mathrm{k}+}$ is introduced into solution, an additional equilibrium, equivalent of (2), is established. Depending on $\mathrm{pH}$ of electrolyte, this second ion will form positive $\left(\mathrm{M}^{\mathrm{k}}\right)$ or negative $\left([\mathrm{MY}]^{(\mathrm{n}-\mathrm{k})}\right)$ charged species. However, stability constant of chelates [MeY] and [MY] in general are different, hence altering between cationic and anionic form for each of the nuclides will be occurred at individual $\mathrm{pH}$ values. Thus, in general, it is always possible to specify a $\mathrm{pH}$ region in which two given nuclides, mixed with a chelating agent, will form an opposite charged species and, consequently, in electric field will show contramigration.

\section{Example}

As an example, an investigation on $\mathrm{In} / \mathrm{Cd}$ separation possibility along with the $\mathrm{In} / \mathrm{Cd}$ separation itself could be demonstrated. It was shown (2) that in presence of DTPA In and $\mathrm{Cd}$ are represented by opposite charged ions ([In-DTPA $]^{2-}$ and $\mathrm{Cd}^{2+}$, respectively) within a certain $\mathrm{pH}$ region. In order to prove this fact, two sets of experiments were set up. Each of them was aimed on investigation of ion (In, respectively Cd) mobility in presence of DTPA at different $\mathrm{pH}$; all other experimental parameters (ionic strength, temperature etc.) were kept constant. Results for both In and Cd ion mobility are plotted on one graphic (Fig. 3) for comparison. It is clear that within area $1,0<\mathrm{pH}<1,5$ In and $\mathrm{Cd}$ species migrate in opposite directions.

After clarification of $\mathrm{In} / \mathrm{Cd}$ separation's condition, a final experiment was done. Into the electrophoretic tube, filled with an appropriate electrolyte, a trace amount of radioac- 
tive $\mathrm{In}^{3+}$ and $\mathrm{Cd}^{2+}$ was introduced simultaneously. The electromigration progress is presented on Fig. 4. At the be- other, without any overlapping; that is, a full separation is realized.

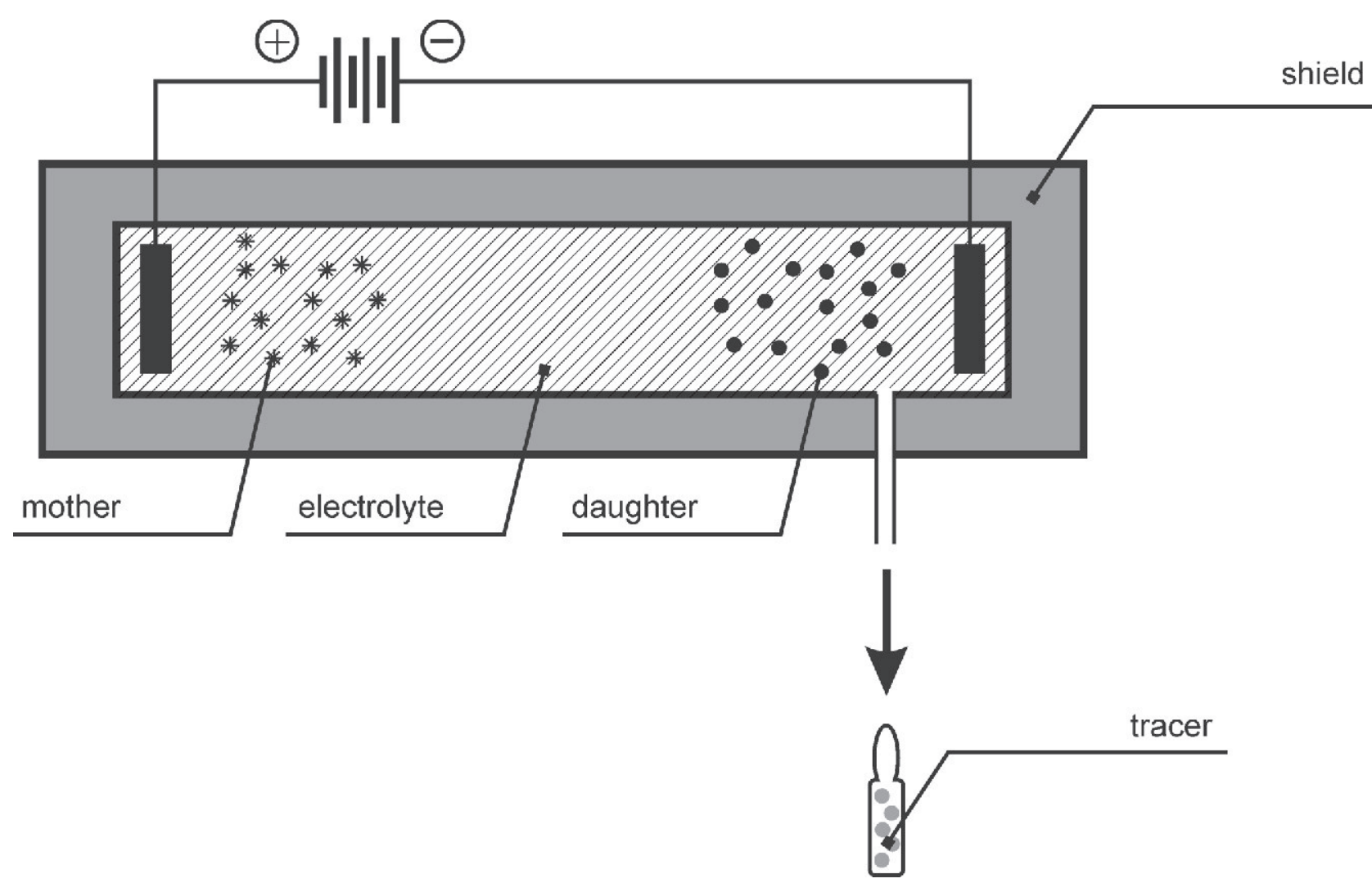

Fig. 2. Schematic construction of new radionuclide generator (electrophoretic type)

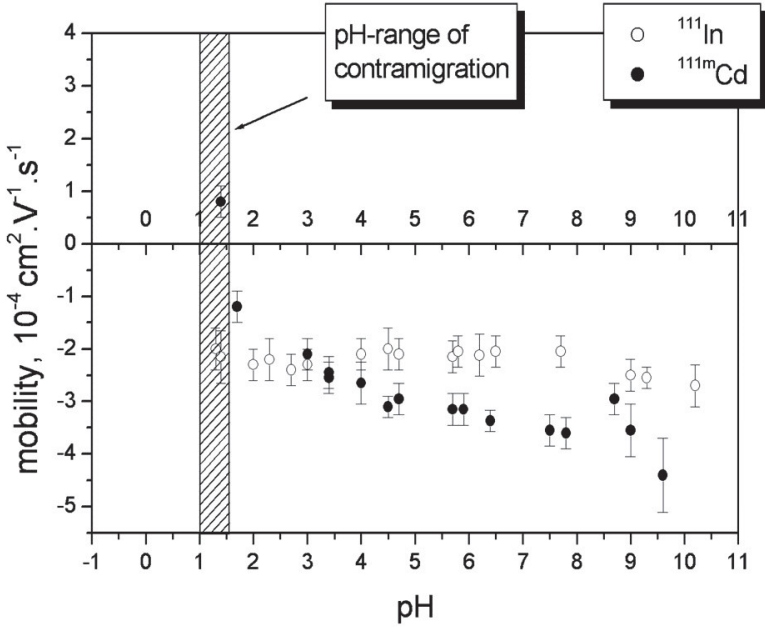

Fig. 3. Comparative analysis of In and Cd ion mobilities vs. pH. Analytical concentration of DTPA 3.10 $0^{-5} \mathrm{M}$, ionic strength $0,100 \mathrm{~mol}^{-1} \mathrm{I}^{-}$, temperature 25,0 ${ }^{\circ} \mathrm{C}$, electric field gradient $10,0 \mathrm{~V} \cdot \mathrm{cm}^{-1}$.

ginning, an united radioactive zone, containing homogenous mixture of $\mathrm{In}$ and $\mathrm{Cd}$ species is observed. After some 12 minutes, due to the opposite electrophoretic behavior of In and $\mathrm{Cd}$ in conditions cited, the corresponding zones are visibly divided already. Twenty minutes later, two migrating zones are detected at considerable distance from each

\section{Effectiveness, limits and comparison}

When considering the medical application of described electrophoretic separation technique, some major questions arise. Firstly, is it always possible to separate any given couple mother/daughter by electrophoresis? The answer is: "generally, yes". Even the most chemically similar cations show differences in their chelate's stability, considerable enough to ensure the existence of corresponding $\mathrm{pH}$-region of contramigration.

Secondly, is it always possible to use gathered solution for direct in-vivo introduction? The answer is again: "generally, yes". Described technique has no requirements about salinity of electrolyte. Separation procedure could be executed in physiological solution, for example. The only parameter to take care of is the $\mathrm{pH}$. In the example given above (separation of couple $\mathrm{In} / \mathrm{Cd}$ ) $\mathrm{pH}$ was chosen to be 1,00 , which is too acidic for direct injection. Therefore it is always possible to select weaker chelating agent, so that $\mathrm{pH}$-region of contramigration shifts toward the neutral $\mathrm{pH}$ area.

And finally, how fast is the electrophoretic separation? Obviously, it depends on type of the ions being separated and gradient of electric field chosen. It could be shown that at realistic $10 \mathrm{~V} . \mathrm{cm}^{-1}$ most ions (average mobility of $3.10^{4}$ $\left.\mathrm{cm}^{2} \cdot \mathrm{V}^{-1} \cdot \mathrm{s}^{-1}\right)$ will fully separate for about 90 seconds. In worst case, when considering well-hydrated, large, heavy-charged ions (mobility down to $1.10^{-4} \mathrm{~cm}^{2} . \mathrm{V}^{-1} \cdot \mathrm{s}^{-1}$ ) separation will take less than 20 minutes. 
There are some distinctive advantages of proposed new electrophoretyc-type generators, which come to live when comparing with existing, chromatographic-type ones. Main advantages could be summarized as follows:
Investigation carried out has led to the conclusion that such a task is completely possible.

The principles of electrophoretic separation conception have been discussed. It has been shown how to track down
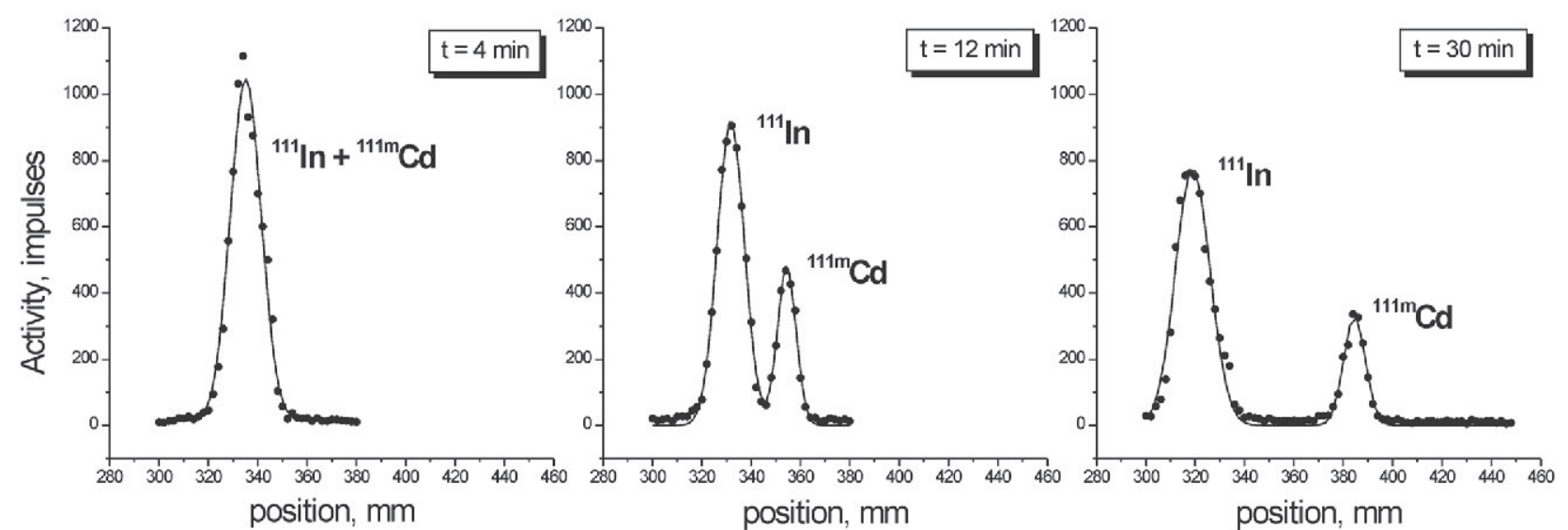

Fig. 4. Electrophoretic separation of $\mathrm{In} / \mathrm{Cd}$ mixture at $\mathrm{pH}=1,00$. Analytical concentration of DTPA 3.10 ${ }^{-5} \mathrm{M}$, ionic strength (perchlorate medium) 0,100 mol. $l^{-1}$, temperature $25,0^{\circ} \mathrm{C}$, electric field gradient $10,0 \mathrm{~V} . \mathrm{cm}^{-1}$.

- Due to the migration in opposite directions, two nuclide forms naturally undergo $100 \%$ separation. Such effectiveness may not be reachable by chromatographic technique;

- In-vivo application could be done immediately after separation procedure. Daughter nuclide is already engaged in suitable complex, forming a ready tracer during the separation itself. No additional kits are required.

- There is zero chance to inject the patient with radioactive solution containing inappropriate form of the nuclide. That is, the separation will take place if only the target nuclide did take the desired complex form. There is no way to forget adding a kit or observe failure in post-separation nuclide-kit incorporation.

- Collected tracer contains the target radionuclide within relatively small volume, e.g. 1-2 ml. That is a welcome fact, because, from practical point of view, it is easier to maintain a high specific activity samples.

- However, some disadvantages of proposed technique should also be mentioned. At first, the method described is inappropriate for dealing with gaseous isotopes. Such a special cases do exist (e.g. the $\mathrm{Rb}-81 / \mathrm{Kr}-81 \mathrm{~m}$ couple), yet are rare ones. At second, the end-user should have a high-voltage DC supply in his disposal. This type of laboratory equipment is relatively standard one though; moreover there are no special technical requirements.

\section{CONCLUSION}

The idea to use the electrophoresis in order to construct a radionuclide generator has been outlined for the first time. the optimal parameters of separation procedure. The existing (chromatographic type) generators, and new, proposed (electrophoretic type) ones have been schematically compared. It has been underlined that some advantages could be expected, such as achieving an extremely high separation rate, avoiding kit usage as well as ensuring the high specific activity of the tracer collected.

Attention has been drawn upon the ease of maintenance (switch on - wait $5 \mathrm{~min}$ - get tracer) as well as the safety of product (zero chance for kit-nuclide engagement failure scenario).

\section{REFERENCES}

1. Bojikov G.A., Ivanov P.I., Priemyshev A.N., Bontchev G.D., Gustova M.V., Starodub G.Y., Milanov M.V., Maslov O.D., Dmitriev S.N., Production and radiochemical separation of ${ }^{88} \mathrm{Zr}$ and ${ }^{175} \mathrm{Hf}$, JINR FLNR Scientific Reports "Heavy Ion Physics" Dubna, Russia, p. 127 (2001)

2. Bontchev G.D., Filossofov D.V., Bojikov G.A., Priemyshev A.N., Ivanov P.I., Maslov O.D., Milanov M.V., Dmitriev S.N.. Electrophoretic separation of In(III) and Cd(II) ions. JINR FLNR Scientific Reports 1999-2000 "Heavy Ion Physics", Dubna (2001), p. 129-130

3. Dmitriev S.N., Milanov M.V., Alikov B. A., Bojikov G.A., Bontchev G.D., Furyaev T.A., Maslov O.D., Priemyshev A.N., Salamatin A.B., Device for horizontal zone electrophoresis in free electrolyte, JINR FLNR Scientific Reports 1997-1998 “Heavy Ion Physics", Dubna, Russia, p. $207(2000)$

4. Filossofov D.V., Lebedev N.A., Novgorodov A.F., Bontchev G.D., Starodub G.Y., Production, concentration and deep purification of ${ }^{111}$ In 
radiochemicals, Appl. Radiat. Isot., 55, p. 293-295 (2001)

5. Ivanov P.I., Bozhikov G.A., Bontchev G.D., Milanov M.V., Maslov O.D., Dmitriev S.N.. Electrophoretic separation of $\mathrm{Zr}(\mathrm{IV})$ and $\mathrm{Hf}(\mathrm{IV})$ ions. JINR FLNR Scientific Reports 2001-2002 "Heavy Ion Physics", Dubna (2003), p. 175-176

6. Priemyshev A. N., Bontchev G.D., Bozhikov G.A., Alikov B.A., Salamatin A.B., Furyaev
T.A., Maslov O.D., Milanov M.V., Dmitriev S.N., Device for horizontal zone electrophoresis in free electrolyte, Preprint JINR P13-2000-43, Dubna, Russia (2000)

7. Radioisotopes in medicine, World nuclear association, Information papers, Bulletin \#INF55 (2008) 